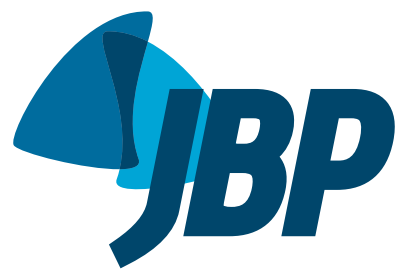

\title{
Diagnostic value of endobronchial ultrasound-guided transbronchial needle aspiration in various lung diseases
}

\author{
Mediha Gonenc Ortakoylu', Sinem Iliaz', Ayse Bahadir' ${ }^{1}$ Asuman Aslan', \\ Raim Iliaz², Mehmet Akif Ozgul' ${ }^{1}$, Halide Nur Urer ${ }^{3}$
}

1. Department of Pulmonology, Istanbul Yedikule Chest Diseases and Thoracic Surgery Training and Research Hospital, Istanbul, Turkey.

2. Department of Internal Medicine Istanbul University Istanbul Medical Faculty, Istanbul, Turkey.

3. Department of Pathology, Istanbul Yedikule Chest Diseases and Thoracic Surgery Training and Research Hospital, Istanbul, Turkey.

Submitted: 11 November 2014 Accepted: 11 June 2015

Study carried out in the Department of Pulmonology, Istanbul Yedikule Chest Diseases and Thoracic Surgery Training and Research Hospital, Istanbul, Turkey.

\begin{abstract}
Objective: Endobronchial ultrasound-guided transbronchial needle aspiration (EBUSTBNA) is a new method for the diagnosis and staging of lung disease, and its use is increasing worldwide. It has been used as a means of diagnosing lung cancer in its initial stages, and there are data supporting its use for the diagnosis of benign lung disease. The aim of this study was to share our experience with EBUS-TBNA and discuss its diagnostic value. Methods: We retrospectively analyzed the results related to 159 patients who underwent EBUS-TBNA at our pulmonary medicine clinic between 2010 and 2013. We recorded the location and size of lymph nodes seen during EBUS. Lymph nodes that appeared to be affected on EBUS were sampled at least twice. We recorded the diagnostic results of EBUS-TBNA and (for cases in which EBUS-TBNA yielded an inconclusive diagnosis) the final diagnoses after further investigation and follow-up. Results: We evaluated 159 patients, of whom 89 (56\%) were male and 70 $(44 \%)$ were female. The mean age was $54.6 \pm 14.2$ years among the male patients and $51.9 \pm 11.3$ years among the female patients. Of the 159 patients evaluated, $115(84 \%)$ were correctly diagnosed by EBUS. The diagnostic accuracy of EBUS-TBNA was 83\% for benign granulomatous diseases and $77 \%$ for malignant diseases. Conclusions: The diagnostic value of EBUS-TBNA is also high for benign pathologies, such as sarcoidosis and tuberculosis. In patients with mediastinal disorders, the use of EBUS-TBNA should be encouraged, primarily because it markedly reduces the need for mediastinoscopy.

Keywords: Sarcoidosis; Tuberculosis, pulmonary; Lung neoplasms; Bronchoscopy; Mediastinoscopy; Endosonography.
\end{abstract}

\section{INTRODUCTION}

Endosonography was initially used in the staging of gastrointestinal tract malignancies. ${ }^{(1)}$ In the 1990 s, it was adapted for use in bronchial diseases. In patients with lung disease, its uses now include tumor staging; the diagnosis of central (parenchymal) masses; and the detection of mediastinal or hilar lymphadenopathy. Endobronchial ultrasound (EBUS) enables the visualization of lymph node structure, thus allowing the pulmonologist to evaluate and sample lymph nodes. Consequently, minimally invasive staging of lung cancer has advanced considerably. In addition, tumor invasion of the tracheobronchial wall can be assessed more accurately with EBUS than with CT. The accuracy of EBUS in making this distinction is $94 \%$, compared with $51 \%$ for CT. ${ }^{(2)}$ Lymph node stations $2,4,7,10$, and 11 can be sampled by EBUS. If EBUS is combined with esophageal ultrasound, lymph node stations 5, 8, and 9 can also be sampled. Therefore, the combination of esophageal ultrasound and EBUS can be seen as the first and best test in patients with suspected lymph node metastasis. ${ }^{(3)}$ There are studies showing that this is a good alternative to mediastinoscopy. ${ }^{(4,5)}$

In patients with malignant disease or granulomatous diseases such as tuberculosis and sarcoidosis, EBUS can contribute to the diagnosis. In a recent meta-analysis, the diagnostic accuracy of EBUS-guided transbronchial needle aspiration (EBUS-TBNA) for sarcoidosis was shown to be $54-93 \%$. ${ }^{(6)}$ In tuberculosis, for which EBUS is also diagnostic, EBUS-TBNA has been shown to have a sensitivity of $85 \%$.(7)

In the evaluation of airway disease, EBUS has emerged as a technique that has great potential for development. Different diagnostic values for EBUS have been reported in various studies. The aim of this study was to determine the diagnostic value of EBUS-TBNA, its contribution to the diagnosis of different diseases, and the factors that determine the magnitude of that contribution.

\section{METHODS}

\section{Patients and procedures}

In this study, we retrospectively analyzed 159 patients in whom EBUS-TBNA was used at our pulmonary medicine clinic between 2010 and 2013. In patients with mediastinal/ hilar lymphadenopathy, EBUS-TBNA was performed in order to evaluate the etiology. Lymphadenopathy was defined as a finding of one or more lymph nodes with a short-axis diameter of $\geq 10 \mathrm{~mm}$ on CT or high 
18F-fluorodeoxyglucose uptake on positron emission tomography/CT. All of the patients evaluated gave written informed consent. Patients fasted for at least $4 \mathrm{~h}$ before the procedure. The preparation process included local anesthesia with lidocaine hydrochloride (Xylocaine ${ }^{\circledR}$ ) and sedation with midazolam just prior to the EBUS procedure, which was performed with a convex-probe ultrasound-guided fiberoptic bronchoscope (BF-UC160F-OL8; Olympus Medical Systems, Tokyo, Japan). All EBUS-TBNA procedures were performed by the same pulmonologist. The location and size of the lymph nodes seen during EBUS were recorded. Lymph nodes that appeared to be affected were sampled at least twice. The samples were sent for histopathological assessment. Rapid onsite cytopathological examination was not performed. If EBUS-TBNA did not lead to a diagnosis, the patients underwent mediastinoscopy, open lung biopsy, or transthoracic needle aspiration (TTNA), according to the situation. In patients with pathological diagnosis of chronic granulomatous inflammation, the diagnosis of tuberculosis or sarcoidosis was determined from the EBUS-TBNA results. This determination was made on the basis of the presence of necrosis in the samples; clinical symptoms; the history of contact with tuberculosis cases; microbiological evaluation; tuberculin skin test results; and additional biochemical features. Samples were classified as containing insufficient material if they contained no lymphocytes. The cytology of a sample was classified as benign (normal) if it presented mature lymphocytes or anthracosis, without malignant cells or granulomas. The final diagnosis was based on the cytology, surgical results, or clinical follow-up. We recorded all diagnoses resulting from EBUS-TBNA. For the patients with inconclusive EBUS-TBNA results, the final diagnoses were recorded as those made after surgery, TTNA, or at least 6 months of follow-up (i.e., bacteriological and clinical outcomes). Patients were grouped by final diagnosis: malignant disease; benign disease; or inconclusive (normal cytology/anthracosis or insufficient material).

\section{Statistical analysis}

For statistical analysis of the data, we used the Statistical Package for the Social Sciences, version 15.0 for Windows (SPSS Inc., Chicago, IL, USA). We used descriptive statistics, including mean, standard deviation, and frequency. The lymph node size that supported the EBUS-TBNA-determined diagnosis was calculated via ROC analysis. Values of $p<0.05$ were considered statistically significant.

\section{RESULTS}

Of the 159 patients evaluated by EBUS-TBNA in the present study, $89(56 \%)$ were male and $70(44 \%)$ were female. The mean age was $54.6 \pm 14.2$ years for the male patients and $51.9 \pm 11.3$ years for the female patients. Patients enrolled in the study were similar in terms of gender and age distribution $(p=0.13)$.
The EBUS-TBNA-determined diagnoses were as follows: sarcoidosis (in 43 patients); tuberculosis (in 14); malignancy (in 33); and normal cytology/anthracosis (in 58). In addition, EBUS-TBNA resulted in one patient being diagnosed with nocardiosis and another being diagnosed with a cyst in the subcarinal area. In 92 of the 159 patients, the EBUS-TBNA procedure was diagnostic. In 9 patients, the EBUS-TBNA samples were classified as containing insufficient material. Further investigation (surgery, TTNA, or follow-up) of those 9 patients revealed one case of sarcoidosis and one case of malignancy, the remaining seven cases being lost to follow-up. In the analysis of the EBUS-TBNA samples, the cytology was categorized as normal in 58 patients. Further evaluation and assessment of this group yielded the following diagnoses: sarcoidosis $(n=6)$; tuberculosis $(n=5)$; malignancy $(n=9)$; nocardiosis $(n=1)$; and normal cytology/anthracosis $(n=22)$. The remaining 15 patients were lost to follow-up, and the final diagnoses were therefore unknown. Of the 159 patients evaluated, 114 (83\%) were correctly diagnosed using EBUS-TBNA. That group included those diagnosed with sarcoidosis or tuberculosis, as well as those in which the cytology was categorized as malignant (true positive) or benign (true negative). Figure 1 details the distribution of the final diagnoses.

Of the 50 patients receiving a final diagnosis of sarcoidosis, $43(86 \%)$ were diagnosed with EBUSTBNA, as were $14(74 \%)$ of the 19 patients receiving a final diagnosis of tuberculosis. In the sarcoidosis and tuberculosis group, when we considered those 69 cases collectively (as the benign granulomatous disease category), EBUS-TBNA had a diagnostic accuracy of $83 \%$. Among the 159 patients analyzed, the final diagnosis was malignancy in 43 . In $33(77 \%)$ of those patients, the diagnosis was based on the EBUS-TBNA findings. Among the remaining 10 patients (i.e., the $23 \%$ that were not diagnosed with EBUS-TBNA), there were seven cases of lung malignancy, two cases of hematologic malignancy (plasmacytoma and lymphoma, respectively), and one case of esophageal cancer. Therefore, for malignancy, EBUS-TBNA had a negative predictive value of $92 \%$ and an accuracy of $94 \%$. The distribution of the final diagnoses and the diagnostic accuracy of EBUS-TBNA for each are given in detail in Table 1. The diagnostic accuracy of EBUS-TBNA was found to be similar for benign and malignant pathologies $(p=0.39$ ). No serious complications (requiring early termination of the procedure) were found to have occurred during the use of EBUS-TBNA.

When we evaluated factors that might affect the diagnostic accuracy of EBUS-TBNA, neither age nor gender were found to have any such effect $(p=0.05$ and $p=0.43$, respectively). The number and size of lymph nodes detected using EBUS-TBNA are given in detail in Table 2. In the presence of enlarged mediastinal (N2) lymph nodes, the likelihood of obtaining a diagnosis with EBUS-TBNA increased significantly $(p=0.013)$. Enlargement of hilar lymph nodes had no such effect 
$(p=0.065)$. The ROC analysis, performed in order to assess the contribution of lymph node size to the likelihood of diagnosis, revealed that, for obtaining an EBUS-TBNA-based diagnosis of granulomatous disease or malignancy, a finding of lymph nodes with a short-axis diameter $\geq 16.5 \mathrm{~mm}$ had a sensitivity of $60 \%$ and a specificity of $76 \%$ (Figure 2 ). For that cut-off value, the positive predictive value was $71 \%$, the negative predictive value was $66 \%$, and the area under the curve was 0.728 . When that lymph node size cut-off value was applied, the diagnostic accuracy of EBUS-TBNA was $69 \%(p<0.001)$.

Table 1. Final diagnoses and diagnostic accuracy of endobronchial ultrasound-guided transbronchial needle aspiration.

\begin{tabular}{lcc}
\hline Type of disease & $\begin{array}{c}\text { Final } \\
\text { diagnosis }\end{array}$ & $\begin{array}{c}\text { Definitive } \\
\text { diagnosis by } \\
\text { EBUS-TBNA }\end{array}$ \\
$\begin{array}{l}\text { Malignant } \\
\quad \text { Non-small cell }\end{array}$ & 43 & $33(77)$ \\
$\quad \begin{array}{l}\text { lung cancer } \\
\text { Small cell lung }\end{array}$ & 7 & $26(81)$ \\
cancer & & $6(86)$ \\
Other & & $1(25)$ \\
Benign & 4 & $59(82)$ \\
$\quad$ Sarcoidosis & 72 & $43(86)$ \\
Tuberculosis & 50 & $14(74)$ \\
Other & 19 & $2(67)$ \\
\hline
\end{tabular}

EBUS-TBNA: endobronchial ultrasound-guided transbronchial needle aspiration. aLymphoma $(n=2)$; plasmacytoma $(n=$ $1)$; and esophageal cancer $(n=1)$. ${ }^{b}$ Nocardiosis $(n=2)$; and cyst $(n=1)$.

\section{DISCUSSION}

Although EBUS-TBNA is a new method, its use in the diagnosis and staging of lung diseases has become increasingly widespread. With EBUS-TBNA, it is possible to sample a lesion while it is being displayed by the ultrasound probe. This method has been used for lung cancer staging. It has also been shown to be diagnostic for benign lung diseases..$^{(3-7)}$ In the present study, we found that the overall diagnostic accuracy of EBUS-TBNA (regardless of the etiology) was $83 \%$. Similarly, Choi et al. ${ }^{(8)}$ reported that EBUS-TBNA had an overall diagnostic accuracy of $83.9 \%$. Evaluating our cases over the last four years, we found that EBUS-TBNA has been well tolerated and has proven to be a reliable diagnostic method with few complications. The purpose of the present study was to share our experience regarding the diagnostic value of EBUS-TBNA.

Table 2. Lymph node number and size detected by endobronchial ultrasound.

\begin{tabular}{|lcc|}
\hline Lymph node & $\mathbf{n}$ & $\begin{array}{c}\text { Short-axis } \\
\text { diameter } \\
\text { mm }^{\mathrm{a}}\end{array}$ \\
\hline $2 \mathrm{R}$ & 4 & $8.25(5-35)$ \\
$2 \mathrm{~L}$ & 0 & $14.5(5-41)$ \\
$4 \mathrm{R}$ & 55 & $10.1(4-50)$ \\
$4 \mathrm{~L}$ & 19 & $17(3-42)$ \\
7 & 101 & $14(6-35)$ \\
$10 \mathrm{R}$ or $11 \mathrm{R}$ & 45 & $15(4-45)$ \\
$10 \mathrm{~L}$ or $11 \mathrm{~L}$ & 57 & \\
Total & 281 & \\
\hline aalues expressed as median (min-max).
\end{tabular}

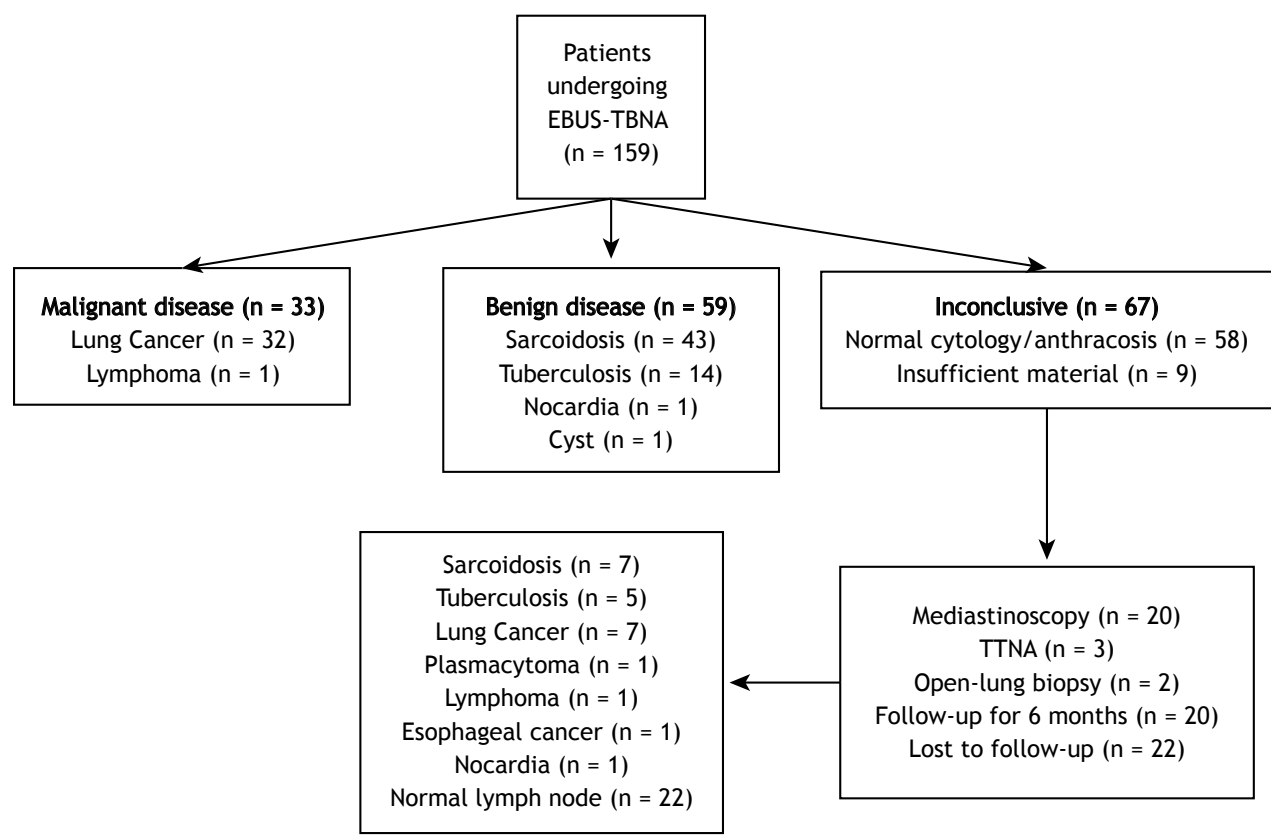

Figure 1. Flow diagram of the diagnoses made by endobronchial ultrasound-guided transbronchial needle aspiration and by other methods. EBUS-TBNA: endobronchial ultrasound-guided transbronchial needle aspiration, TTNA: transthoracic needle aspiration. 
In the diagnosis of sarcoidosis, EBUS-TBNA is often used, because it can preclude the need for conventional TBNA or, in some cases, mediastinoscopy. ${ }^{(4,5,9)}$ The use of EBUS-TBNA guarantees that the sample was taken from the targeted lymph nodes. Therein lies its superiority over conventional TBNA. ${ }^{(10,11)}$ In addition, EBUS-TBNA is not as invasive as is mediastinoscopy. Various levels of diagnostic accuracy in sarcoidosis have been reported for EBUS-TBNA. A recent meta-analysis found that the reported diagnostic accuracy of EBUSTBNA in sarcoidosis ranged from $54 \%$ to $93 \%$. $^{(6)}$ In our study, that level was $86 \%$, which is in agreement with the findings of other studies in the literature. Navani et al. ${ }^{(12)}$ evaluated patients with suspected sarcoidosis using EBUS-TBNA and reported a diagnostic accuracy of $88 \%$. When those same patients underwent transbronchial lung biopsy, mucosal biopsy, and BAL via standard bronchoscopy, the diagnostic accuracy increased to $93 \%$. The variability in the reported levels of diagnostic accuracy across studies might reflect the level of experience of the pulmonologist performing the EBUS-TBNA or that of the pathologist, as well as the difference between studies in which rapid onsite cytopathological examination was performed and those in which it was not.

In another benign disease, tuberculosis, EBUSTBNA is also used as a diagnostic method. Sun et al. ${ }^{(7)}$ and Cetinkaya et al. ${ }^{(13)}$ reported the sensitivity of EBUS-TBNA in tuberculosis to be $85 \%$ and $79 \%$, respectively, ${ }^{(7,13)}$ compared with the $74 \%$ found in the present study. The diagnostic accuracy of EBUS-TBNA is lower for tuberculosis than for sarcoidosis, and conventional TBNA has a diagnostic accuracy of $65 \%$ for tuberculosis. ${ }^{(14)}$ Ren et al. ${ }^{(15)}$ found that, in patients with suspected mediastinal tuberculous lymphadenitis, standard bronchoscopy had a sensitivity of $18.1 \%$

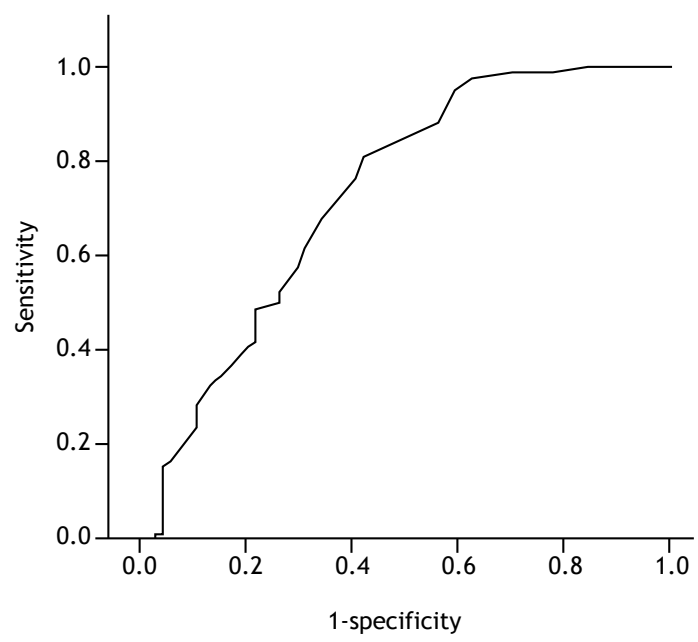

Figure 2. ROC curve analysis: when a lymph node size (short-axis diameter) cut-off value of $16.5 \mathrm{~mm}$ was applied, the sensitivity and specificity of endobronchial ultrasound (for a diagnosis of granulomatous disease or malignancy) were $76 \%$ and $60 \%$, respectively. Diagonal segments are produced by ties. and a specificity of $100 \%$. When the authors added EBUS-TBNA to standard bronchoscopy, those values rose to $80 \%$ and $92.3 \%$, respectively. ${ }^{(15)}$ Therefore, EBUS-TBNA seems to contribute to the diagnostic accuracy in patients with tuberculous lymphadenitis and mediastinal lymph node enlargement. In the present study, we also found the diagnostic accuracy of EBUSTBNA to be lower for tuberculosis than for sarcoidosis. The prominent necrosis in paucibacillary tuberculosis could explain that finding. Dhooria et al. ${ }^{(16)}$ reported that one can distinguish between tuberculosis and sarcoidosis on the basis of EBUS images. Accordingly, when combined with a positive tuberculin skin test result, the heterogeneous echotexture of the internal structure or necrotic appearance seen via EBUS can diagnose tuberculosis with a specificity of $98 \%$ and a positive predictive value of $91 \%$. $^{(16)}$

One recent study reported that EBUS-TBNA has a diagnostic accuracy of $93.9 \%$ for malignancy, ${ }^{(8)}$ compared with only $77 \%$ in the present study. That same study reported that the diagnostic accuracy of EBUS-TBNA was higher for malignant diseases than for benign diseases (93.9\% vs. 70.6\%, p < 0.001). In our study, the diagnostic accuracy of EBUS-TBNA for benign granulomatous disorders was not significantly different from that observed for malignant disease ( $83 \%$ vs. $77 \%, p>0.05$ ). Memoli et al. ${ }^{(17)}$ showed that increased lymph node size supports an EBUS-based diagnosis of malignancy. Abu-Hijleh et al. ${ }^{(18)}$ suggested that lymph node size has little effect on diagnostic accuracy or sensitivity of EBUS-TBNA. In contrast, when lymph node size exceeds $20 \mathrm{~mm}$, the negative predictive value decreases. Those authors found that samples taken from lymph nodes $\geq 20 \mathrm{~mm}$ in size always contained sufficient material for analysis. The same authors reported that samplings of the $4 R, 4 L$, $7,10 / 11 \mathrm{R}$, and $10 / 11 \mathrm{~L}$ lymph nodes did not differ in terms of diagnostic accuracy. ${ }^{(18)}$ Tedde et al. ${ }^{(19)}$ showed that identifying lymphadenopathy in multiple lymph nodes and sampling subcarinal lymph nodes provides a higher diagnostic yield in EBUS-TBNA. Those authors reported that EBUS-TBNA has a diagnostic accuracy of $57 \%$ for malignancy. In the present study, lymph nodes with a short-axis diameter $\geq 16.5 \mathrm{~mm}$ were found to be more diagnostic in the histopathological evaluation than were those with smaller diameters. We also found that the diagnostic accuracy of EBUS-TBNA was better when there was mediastinal lymph node enlargement than when there was hilar lymph node enlargement. In a study conducted by Cetinkaya et al.,(20) the sensitivity of EBUS-TBNA was lower in the region of lymph node $4 \mathrm{~L}$ than in those of the other lymph nodes. The authors reported that the overall diagnostic accuracy of EBUS-TBNA was not affected by the number of lymph nodes sampled, the number of times a lymph node region was sampled, or lymph node size. ${ }^{(20)}$

In our study sample, we noted that EBUS-TBNA provoked only minor complications, such as mild hypoxia, tachycardia, and minor hemorrhage. None of 
those complications changed the course of the procedure or led to additional treatment or hospitalization. We can therefore assert that the procedure can be used safely in all adult age groups. Similar studies in the literature have also reported that EBUS-TBNA is safe and causes no severe complications. ${ }^{(8,20-23)}$

In conclusion, EBUS-TBNA is a proven method in the diagnosis and staging of malignancy. The diagnostic accuracy of this method is also high in benign pathologies such as sarcoidosis and tuberculosis. It is a method that is well-tolerated and minimally invasive, with a low rate of complications and high diagnostic accuracy. The use of EBUS-TBNA should be encouraged as the first procedure of choice for mediastinal and hilar lung pathologies, because it markedly reduces the need for mediastinoscopy.

\section{ACKNOWLEDGMENTS}

We are grateful to Mr. David Chapman for editing the language of the manuscript.

\section{REFERENCES}

1. Ziegler K, Sanft C, Semsch B, Friedrich M, Gregor M, Riecken EO. Endosonography is superior to computed tomography in staging tumors of the esophagus and cardia. Gastroenterology 1988;94(Suppl):A517.

2. Herth F, Ernst A, Schulz M, Becker $H$. Endobronchial ultrasound reliably differentiates between airway infiltration and compression by tumor. Chest. 2003;123(2):458-62. http://dx.doi.org/10.1378/ chest.123.2.458

3. Silvestri GA, Gonzales AV, Jantz MA, Margolis ML, Gould MK Tanoue LT, et al. Methods for staging non-small cell lung cancer: Diagnosis and management of lung cancer, 3rd ed. American College of Chest Physicians evidence-based clinical practice guidelines. Chest. 2013:143(5 Suppl):e211S-50S

4. Annema JT, van Meerbeeck JP, Rintoul RC, Dooms C, Deschepper E, Dekkers OM, et al. Mediastinoscopy vs endosonography for mediastinal nodal staging of lung cancer: a randomized trial. JAMA 2010; 304(20): 2245-52. http://dx.doi.org/10.1001/jama.2010.1705

5. Yasufuku K, Pierre A, Darling G, de Perrot M, Waddell $T$ Johnston $M$, et al. A prospective controlled trial of endobronchia ultrasound-guided transbronchial needle aspiration compared with mediastinoscopy for mediastinal lymph node staging of lung cancer J Thorac Cardiovasc Surg. 2011;142(6):1393-400.e1. http://dx.doi. org/10.1016/j.jtcvs.2011.08.037

6. Agarwal R, Srinivasan A, Aggarwal AN, Gupta D. Efficacy and safety of convex probe EBUS-TBNA in sarcoidosis: a systematic review and meta-analysis. Respir Med. 2012;106(6):883-92. http://dx.doi org/10.1016/j.rmed.2012.02.014

7. Sun J, Teng J, Yang H, Li Z, Zhang J, Zhao H, et al. Endobronchial ultrasound-guided transbronchial needle aspiration in diagnosing intrathoracic tuberculosis. Ann Thorac Surg. 2013; 96(6):2021-7. http://dx.doi.org/10.1016/j.athoracsur.2013.07.005

8. Choi YR, An JY, Kim MK, Han HS, Lee KH, Kim S, et al. The diagnostic efficacy and safety of endobronchial ultrasound-guided transbronchia needle aspiration as an initial diagnostic tool. Korean $\mathrm{J}$ Intern Med. 2013;28(6):660-7. http://dx.doi.org/10.3904/kjim.2013.28.6.660

9. Yasufuku K. Current clinical applications of endobronchial ultrasound Expert Rev Respir Med. 2010;4(4):491-8. http://dx.doi.org/10.1586/ ers.10.39

10. Yasufuku K, Chiyo M, Sekine Y, Chhajed PN, Shibuya K, lizasa T, et al. Real-time endobronchial ultrasound-guided transbronchial needle aspiration of mediastinal and hilar lymph nodes. Chest. 2004;126(1):122-8. http://dx.doi.org/10.1378/chest.126.1.122

11. Herth FJ, Eberhardt R, Vilmann P, Krasnik M, Ernst A. Real-time endobronchial ultrasound guided transbronchial needle aspiration for sampling mediastinal lymph nodes. Thorax. 2006;61(9):795-8. http:// dx.doi.org/10.1136/thx.2005.047829

12. Navani N, Booth HL, Kocjan G, Falzon M, Capitanio A, Brown JM, et al. Combination of endobronchial ultrasound-guided transbronchia needle aspiration with standard bronchoscopic techniques for the diagnosis of stage | and stage || pulmonary sarcoidosis. Respirology. 2011;16(3):467-72. http://dx.doi.org/10.1111/j.1440- 1843.2011.01933.x

13. Cetinkaya E, Gunluoglu G, Ozgul A, Gunluoglu MZ, Ozgul G, Seyhan $E C$, et al. Value of real-time endobronchial ultrasound-guided transbronchial needle aspiration. Ann Thorac Med. 2011;6(2):77-81. http://dx.doi.org/10.4103/1817-1737.78422

14. Cetinkaya E, Yildiz P, Altin S, Yilmaz V. Diagnostic value of transbronchial needle aspiration by Wang 22-gauge cytology needle in intrathoracic lymphadenopathy. Chest. 2004; 125(2):527-31. http:// dx.doi.org/10.1378/chest.125.2.527

15. Ren S, Zhang Z, Jiang H, Wu C, Liu J, Liang L, et al. Combination of endobronchial ultrasound-guided transbronchial needle aspiration with standard bronchoscopic techniques enhanced the diagnosis yields of pulmonary tuberculosis patients with lymphadenopathy. Panminerva Med. 2013;55(4):363-70.

16. Dhooria S, Agarwal R, Aggarwal AN, Bal A, Gupta N, Gupta D Differentiating tuberculosis from sarcoidosis by sonographic characteristics of lymph nodes on endobronchial ultrasonography: a study of 165 patients. J Thorac Cardiovasc Surg. 2014;148(2):662-7. http://dx.doi.org/10.1016/j.jtcvs.2014.01.028

17. Memoli JS, El-Bayoumi E, Pastis NJ, Tanner NT, Gomez M, Huggins JT, et al. Using endobronchial ultrasound features to predict lymph node metastasis in patients with lung cancer. Chest. 2011:140(6):1550-6. http://dx.doi.org/10.1378/chest.11-0252

18. Abu-Hijleh M, El-Sameed Y, Eldridge K, Vadia E, Chiu H, Dreyfuss $Z$, et al. Linear probe endobronchial ultrasound bronchoscopy with guided transbronchial needle aspiration (EBUS-TBNA) in the evaluation of mediastinal and hilar pathology: introducing the procedure to a teaching institution. Lung. 2013;191(1):109-15. http:// dx.doi.org/10.1007/s00408-012-9439-z

19. Tedde ML, Figueiredo VR, Terra RM, Minamoto $H$, Jatene FB Endobronchial ultrasound-guided transbronchial needle aspiration in the diagnosis and staging of mediastinal lymphadenopathy: initia experience in Brazil. J Bras Pneumol. 2012;38(1):33-40

20. Cetinkaya E, Ozgül MA, Tutar N, Ozgül G, Cam E, Bilaçeroglu S. The diagnostic utility of real-time EBUS-TBNA for hilar and mediastinal lymph nodes in conventional TBNA negative patients. Ann Thorac Cardiovasc Surg. 2014;20(2):106-12. http://dx.doi.org/10.5761/atcs. oa.12.02072

21. Gurioli C, Ravaglia C, Romagnoli M, Casoni G, Tomassetti S, Nann $O$, et al. EBUS-TBNA in mediastinal/hilar lymphadenopathies and/or masses: an Italian case series. Clin Respir J. 2012;6(1):3-8. http:// dx.doi.org/10.1111/j.1752-699X.2010.00232.x

22. Varela-Lema L, Fernández-Villar A, Ruano-Ravina A. Effectiveness and safety of endobronchial ultrasound-transbronchial needle aspiration: a systematic review. Eur Respir J. 2009;33(5):1156-64. http://dx.doi.org/10.1183/09031936.00097908

23. Gu $P$, Zhao $Y Z$, Jiang $L Y$, Zhang $W$, Xin $Y$, Han BH. Endobronchia ultrasound-guided transbronchial needle aspiration for staging of lung cancer: a systematic review and meta-analysis. Eur J Cancer. 2009;45(8):1389-96. http://dx.doi.org/10.1016/j.ejca.2008.11.043 